MICROSTRUCTURE CROSSCORRELATION IN PULSES SIMULTANEOUSLY OBSERVED AT FREQUENCIES SEPARATED BY UP TO $1 \mathrm{GHz}$

\author{
Valentin Boriakoff \\ National Astronomy and Ionosphere Center \\ Corne11 University, Ithaca, NY 14853 \\ Dale C. Ferguson \\ NAIC, Arecibo Observatory \\ Arecibo, Puerto Rico 00612
}

\title{
ABSTRACT
}

Microstructure crosscorrelation of pulses observed simultaneously at various separations in frequency in the range of 196 to $1420 \mathrm{MHz}$ was done for PSR $0950+08$ and PSR $1133+16$ at the Arecibo Observatory. Microstructure and subpulse components were identified in the crosscorrelations, and their behaviour vs. pulse longitude was studied. Drastic differences in the subpulse and micropulse behaviour are noted, related in particular to component separation in the double peak average pulse profile of PSR 1133+16. Here the subpulses follow the average pulse profile component separation but the microstructure does not. Interpretation and constraints in terms of some current theories are also presented.

\section{OBSERVATIONS AND RESULTS}

The pulsars PSR $0950+08$ and PSR $1133+16$ were observed simultaneously at Arecibo Observatory at pairs of frequencies: at 196.3 and $318 \mathrm{MHz}$, 530 and $1020 \mathrm{MHz}$ and 430 and $1406 \mathrm{MHz}$ with 25 and $40 \mu \mathrm{s}$ sampling time resolutions (equivalent time constants of 60 to $130 \mu \mathrm{s}$ ). Only linear polarization was used, both parallel and orthogonal. The signals were dedispersed by a post-detection hardware dedisperser (Boriakoff 1973, 1974) with filter banks of $32 \times 0.25 \mathrm{MHz}$ and $32 \times 0.02 \mathrm{MHz}$ filters. Crosscorrelation (ccf) and autocorrelation (acf) of signals show the following properties of pulses:

(This first part was done by one of us [V. Boriakoff].) 1) In PSR $1133+16(318-196.2 \mathrm{MHz})$ the two peaks of the average pulse profile separate from the fiducial point (center between the two peaks) with a $v^{-0.26}$ function of frequency (Craft 1970). Subpulses were found to have two different acf characteristic times, $3 \mathrm{~ms}$ and (previously un- 


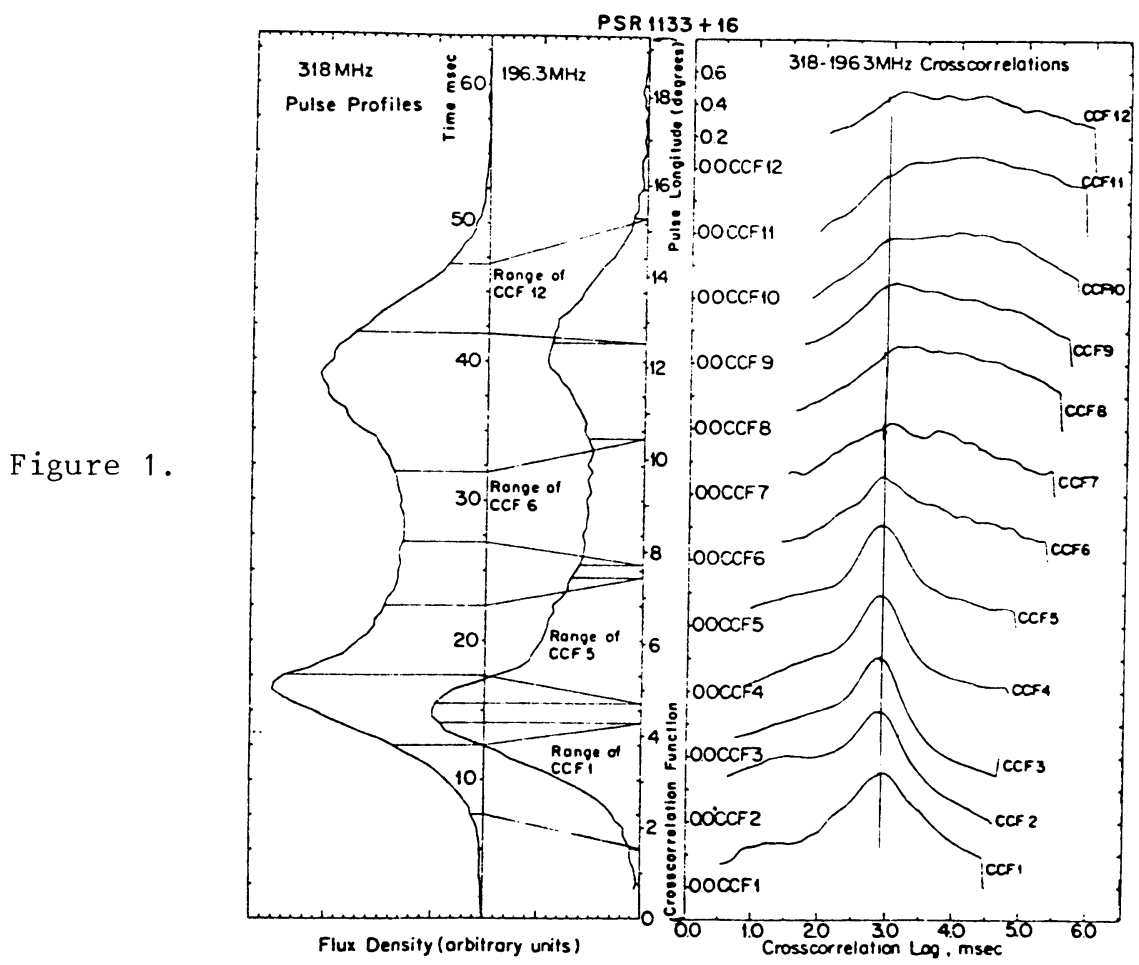

known) $1.5 \mathrm{~ms}$. Both subpulse features follow the separation of the average pulse profile peaks with frequency. Fig. 1 shows in the right side ccf's of small data windows ( $1: 52$ of pulse longitude done at different longitudes of the average pulse profile). The narrow feature (580 $\mu \mathrm{s}$ breakpoint) best seen in CCF 1-5 happens to be vertically aligned, that is, the features causing it (microstructure) always have the same delay from 318 to $196.3 \mathrm{MHz}$ independent of longitude. This delay was used to align the origins of the two average pulse profiles shown in the left side of Fig. 1. That is, a micropulse appearing at a certain longitude of the $318 \mathrm{MHz}$ average pulse profile will appear directly under it in the $196.3 \mathrm{MHz}$ profile. It so happens that this origin offset brings into alignment the fiducial points of the pulses (the center between the two peaks) at the two frequencies, which follows the dispersion relation (Craft 1970). Hence, the microstructure is simultaneously emitted at the two radio frequencies at the source. The $1.5 \mathrm{~ms}$ subpulse feature is also seen in the ccf's and shows a variable displacement with the location of the data window, which matches the $\nu^{-0.26}$ separation of the average pulse profile peaks when the data windows are at the peak positions. For other subpulse longitudes this displacement is roughly proportional to the distance from the fiducial point. The $3 \mathrm{~ms}$ subpulse feature has been shown by Taylor et al. (1975) to follow the $\nu^{-0.26}$ separation of the average pulse profile peaks as wel1. Acf's confirm the existence of both subpulse time scales by the presence of both breakpoints, and also show characteristic times of 
$550 \mu \mathrm{s}$ (at both 318 and 196.3 MHz) of microstructure. Microstructure is nearly absent in the second pulse peak at $318 \mathrm{MHz}$, but is present at the second peak at $196.3 \mathrm{MHz}$. A steeper spectrum than for the micropulses of the first peak is probably the cause. Microstructure can occasionally have a second breakpoint at $900 \mu \mathrm{s}$. The microstructure crosscorrelation is high $(0.84)$ and its spectrum seems to be steeper than that of subpulses.

The following work was done by both authors.

2) PSR $1133+16 \mathrm{ob}-$ served at 430 and $1020 \mathrm{MHz}$ with orthogonal linear polarizations shows similar results (Figure 2). The ccf and acf's were done over the whole

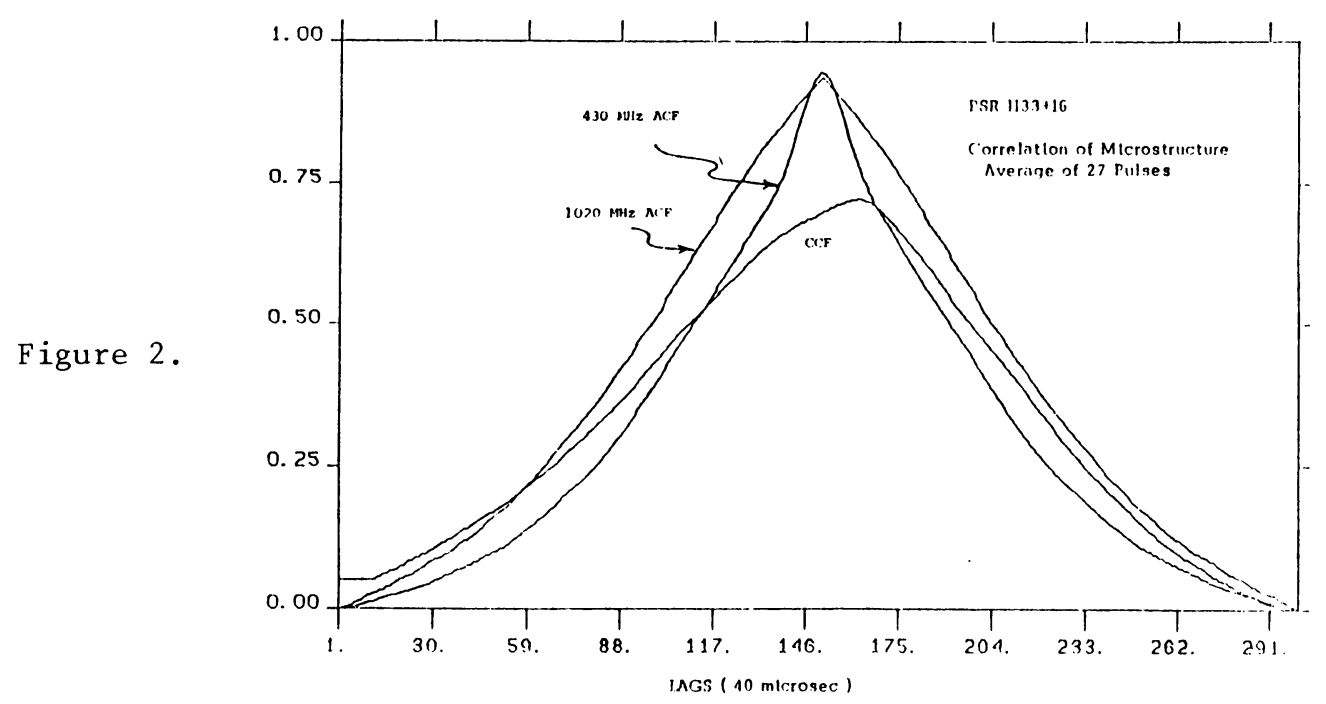

pulse, hence the subpulse part of the ccf is wider. There is a microstructure feature in the ccf which is offset from the center of the subpulse feature. The $430 \mathrm{MHz}$ acf (noise contributions subtracted) is similar to the lower frequency acf's, with similar breakpoints (500 $\mu \mathrm{s}$ and $2.6 \mathrm{~ms}$ ). The $1020 \mathrm{MHz}$ acf is peculiar: the microstructure contribution is small. In fact, the acf is dominated by the subpulse feature. After subtraction of a fitted gaussian from the $430 \mathrm{MHz}$ acf and the ccf (to remove to a large extent the subpulse) we compare the remaining feature and see that the microstructure feature has a similar characteristic time in both. Hence, at $1020 \mathrm{MHz}$ the microstructure that crosscorrelates has a $500 \mu \mathrm{s}$ characteristic time.

3) In Figure 3 we present the PSR 1133+16 430-1406 MHz noisecorrected average acf's and ccf, obtained with parallel linear polarizations. The two whole pulse acf's are similar in shape, their microstructure contributions are similar, but it is difficult to judge the characteristic time of the $1406 \mathrm{MHz}$ microstructure. To be noted is that 


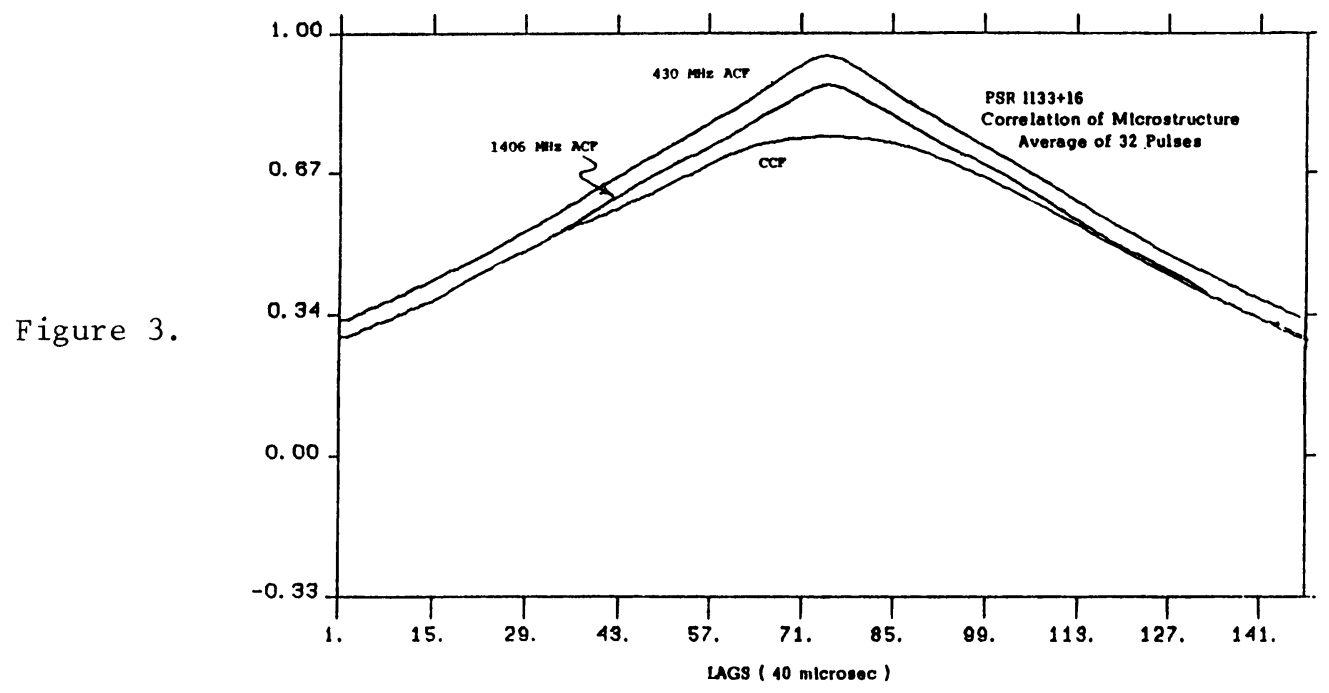

the $1406 \mathrm{MHz}$ microstructure is substantially stronger than the $1020 \mathrm{MHz}$ microstructure. However, the ccf has no microstructure feature. That is, the microstructure at the two frequencies is uncorrelated.

Ferguson and Seiradakis (1978) computed the acf's at 1720 and 2650 $\mathrm{MHz}$; they found strong microstructure features with breakpoints at 380 and $420 \mu \mathrm{s}$ respectively, narrower than the $500 \mu \mathrm{s}$ at $430 \mathrm{MHz}$. No average low frequency $(<1 \mathrm{GHz})$ observations show a narrower feature than $500 \mu \mathrm{s}$. One possibility for the absence of microstructure in the 430-

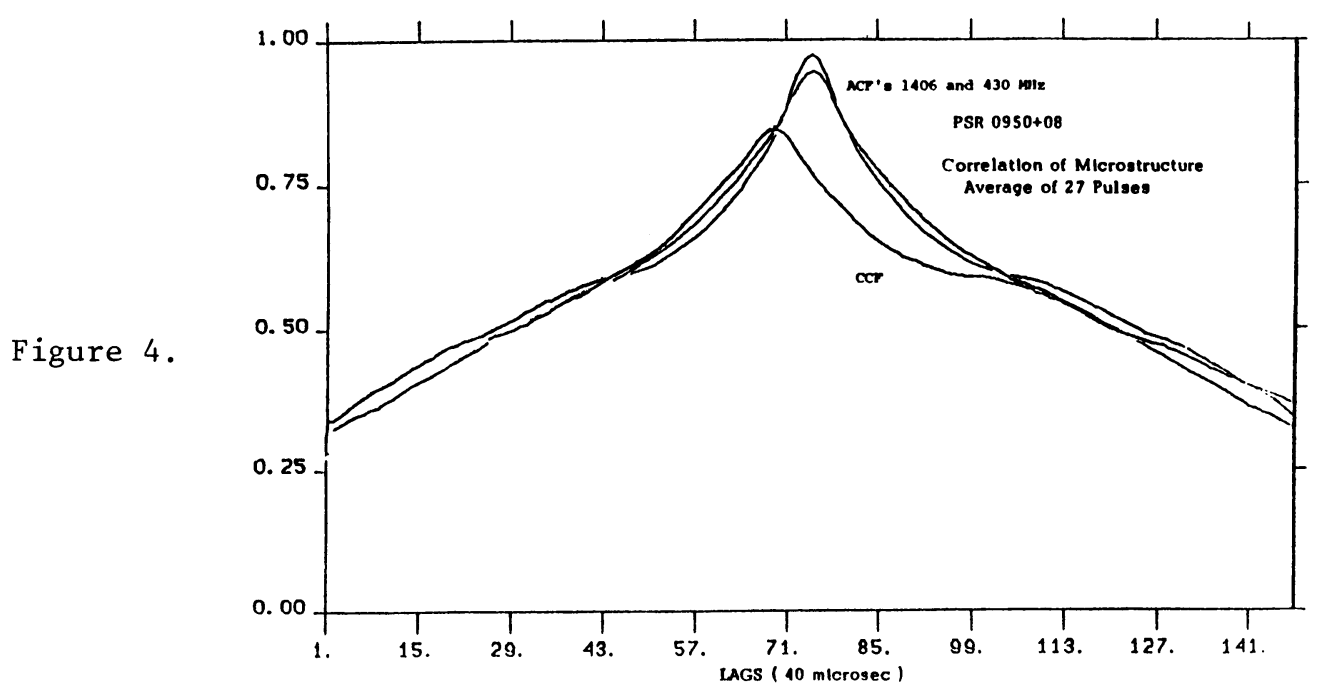


$1406 \mathrm{MHz}$ ccf is Faraday rotation. We do not believe it to be the cause.

4) PSR 0950+08 was observed with a 430-1406 MHz parallel linear polarization system. Figure 4 shows the two noise-corrected acf's, both having similar microstructure components. The ccf has a very prominent microstructure feature, similar in shape and characteristic time to that of the acf's. As with PSR $1133+16$ the ccf microstructure feature is located asymmetrically with respect to the subpulse feature. This quite probably is a consequence of the same effect (at source the microstructure emission is simultaneous at different radio frequencies and the subpulses are not). The microstructure crosscorrelation is high, $\sim 0.66$. Single pulses at both frequencies are of ten very similar in appearance.

\section{CONCLUSIONS}

PSR $1133+16$ apparently has two emission mechanisms: a broadband for microstructure and a narrowband for subpulses. There is a strong indication that all microstructure appearing at frequencies below $v_{\mathrm{c}}$ $\left(1020 \mathrm{MHz}<\nu_{\mathrm{c}}<1406 \mathrm{MHz}\right.$ ) has a characteristic time of $\sim 500 \mu \mathrm{s}$ (independent of frequency) and it does not crosscorrelate with frequencies above $\nu_{c}$, where a smaller characteristic time is seen. Since $\nu_{c}$ is close to the frequency where the frequency dependence of the separation of the average pulse profile of PSR $1133+16$ changes from $\nu^{-0.26}$ to $v^{0.0}$ (Sieber et al. 1975) and to the frequency where the pulse spectral index changes from -1.0 (low frequency) to -2.2 (high frequency) (Sieber 1973) a change in the microstructure emitting mechanism may be responsible. However, subpulses correlate well over a frequency range of 327-2695 MHz (Bartel and Sieber 1978). The similarity of the ccf and acf's implies that all the microstructure wider than about $160 \mu \mathrm{s}$, irrespective of its individual micropulse characteristic width, has the same bandwidth.

In terms of the polar cap model Buschauer and Benford (1980) have developed a physical representation of radio emission where they examine broadband and narrowband emission. Boriakoff (1980) proposes the identification of the narrowband emission with subpulses and broadband with micropulses, but several details should still be explained: why there is a $1 \mathrm{GHz}$ break in the microstructure, why the separation of the average pulse profile peaks changes at $1 \mathrm{GHz}$, how is microstructure generated at $\nu>1.4 \mathrm{GHz}$ that has no low frequency counterpart, etc. PSR $0950+08$ has at least a $1 \mathrm{GHz}$ microstructure bandwidth, so that a very wideband generating process is necessary.

In the velocity of light cylinder model the emission is beamed towards us by the corotation of the emitting bunches. The different time constants of the pulse features are explained by bunching at different radiae (and hence different $\gamma$ ). The constant width of microstructure vs. frequency is explained by having many bunches with different emitted fundamental frequencies (only a narrow frequency band- 
width per bunch) in the same small emission region. In subpulse emission, on the other hand, the bunches emitting different frequencies must be somewhat separated in space, to account for the change of subpulse properties with frequency. Much work needs to be done to model successfully the details of our observations.

This work was supported by the National Astronomy and Ionosphere Center, which is operated by Cornell University under contract from the National Science Foundation.

\section{REFERENCES}

Bartel, N. and Sieber, W.: 1978 , Astron. Astrophys. 70, pp. 307-310. Boriakoff, V.: 1973, "Pulsar Radio Frequency Observations with a Digital Pulsar Processor", NAIC Publication No. 38, Corne11 University, Ithaca, NY.

Boriakoff, V.: 1974, Astron. Astrophys. Supp1. 15, pp. 479-481. Boriakoff, V.: 1980, submitted.

Buschauer, R. and Benford, G.: 1980, Mon. Not. R. astr. Soc. 190, pp. 945-959.

Craft, H.D.: 1970, "Radio Observations of the Pulse Profiles and Dispersion Measures of Twelve Pulsars", Center for Radiophysics and Space Research Publication No. 395, Cornell University, Ithaca, NY.

Ferguson, D.C. and Seiradakis, J.H.: 1978, Astron. Astrophys. 64, pp. $27-42$.

Sieber, W.: 1973, Astron. Astrophys. 28, pp. 237-252.

Sieber, W., Reinecke, R., and Wielebinski, R.: 1975, Astron. Astrophys. 38, pp. 169-182.

Taylor, J.H., Manchester, R.N., and Huguenin, G.R.: 1975, Astrophys. J. 195 , pp. 513-528. 